\title{
Actitudes de los Profesores de Ciencias Naturales y Ciencias Sociales hacia la Enseñanza de Competencias de Consulta en Línea y sus Factores de Fondo en el Uso del Internet
}

\section{The Attitudes of Teachers of Natural and Social Sciences towards Teaching Online Inquiry Competencies and their Underlying Factors in the Use of the Internet}

\footnotetext{
Mariano Eliseo Rodríguez Malebrán

Mario Roberto Quintanilla-Gatica ${ }^{2}$

Miguel Angel Manzanilla ${ }^{3}$
}

\author{
'Universidad de La Serena, La Serena, Chile. Autor Correspondiente: mariano.rodriguez@userena.cl \\ 2Pontificia Universidad Católica de Chile, Facultad de Educación, Santiago, Chile. \\ ${ }^{3}$ Universidad de Los Andes, Facultad de Humanidades y Educación, Mérida, Venezuela.
}

Resumen: La actitud del profesorado es uno de los factores que inciden en la integración de las nuevas tecnologías en el aula. Desde allí nos preguntamos ¿Cómo influyen las actitudes del profesorado de ciencias naturales y ciencias sociales hacia la enseñanza de competencias de consulta en línea y sus factores de fondo en el uso del Internet? Participaron 33 profesores de 18 colegios públicos de una ciudad de Chile, asociados al proyecto conjunto entre Chile y Finlandia, AKA EDU03, aplicando un cuestionario Likert. Luego de un análisis de correspondencias múltiple, la edad se mostró como factor asociado a las actitudes de los profesores para la enseñanza de competencias de consulta en línea, lo que constituye un hallazgo interesante para profundizar en investigaciones futuras, particularmente hoy en actitudes relacionadas con la pandemia COVID 19 que demanda un mayor esfuerzo del profesorado por el uso de tecnologías en un formato remoto de enseñanza, evaluación y aprendizaje.

Palabras clave: Competencia tecnológica; Alfabetización en investigación científica; Enseñanza primaria; Enseñanza de ciencias naturales.

Abstract: The attitude of the teaching staff is one of the factors affecting the integration of new technologies into the classroom. With that in mind, we ask ourselves: how do the attitudes of teachers of natural and social sciences towards teaching online consultation skills and their underlying factors influence the use of the Internet? 33 teachers from 18 public schools in a city of Chile, associated with the joint project between Chile and Finland, AKA EDU03, participated, applying a Likert questionnaire. After a multiple correspondence analysis, age was shown to be a factor associated with teachers' attitudes to teaching online consultation skills, which is an interesting finding for further research, particularly today in attitudes related to the COVID 19 pandemic, which demands greater effort from teachers in using technologies in remote teaching, assessment and learning formats.

Keywords: Technological competence; Scientific research literacy; Primary school; Natural science teaching.

Recebido em: 16/05/2020

Aprovado em: 12/10/2020 


\section{Introducción}

En todo el mundo los medios que se utilizan cada día varían, no obstante es evidente que las tecnologías de la información y la comunicación (TIC), como Internet, representan una influencia importante en la sociedad actual (PUTMAN, 2014). Internet representa una poderosa herramienta de aprendizaje cuando se utiliza adecuadamente, pero su uso eficaz en la educación requiere del análisis de otros factores no sólo desde la herramienta tecnológica (CABERO, 2014). El acceso a la información a través de las nuevas tecnologías necesita como pilar fundamental habilidades de búsqueda y acceso a la información. Por ende, es necesario que los profesores desarrollen las competencias de consulta en línea de los estudiantes para hacer frente a los nuevos retos que plantea el siglo XXI (PRENDES; GUTIÉRREZ, 2013), ya que investigaciones recientes muestran que un número significativo de estudiantes en diferentes niveles, no dominan las habilidades necesarias para desarrollar competencias de consulta en línea eficaces, tales como la localización de la información, la evaluación de la información y la utilización de múltiples fuentes en línea (KIILI; LAURINEN; MARTTUNEN, 2009; SORMUNEN; LEHTIÖ, 2011; LEU et al., 2015).

A su vez, profesores de aula e investigadores, confirmarían que los estudiantes demuestran una mayor motivación y compromiso cuando se les ofrece la oportunidad de utilizar Internet (PUTMAN, 2014). La investigación empírica es necesaria para comprender todo el alcance de cómo estas variables afectivas se manifiestan en la práctica para la enseñanza de competencias de consulta en línea. Sin embargo, no existen instrumentos válidos que midan constructos afectivos en el profesorado, y su relación con los procesos asociados a la enseñanza competencias de consulta en línea (PUTMAN, 2014).

No obstante, algunos instrumentos aplicados a estudiantes tienen relación sobre constructos afectivos que influyen en las competencias de consulta en línea. O’Byrne y McVerry (2008, p. 364, nuestra traducción) desarrollaron un instrumento para medir disposiciones de lectura en línea, que definieron como "[...] actitudes y creencias que conducen a patrones de comportamiento que promueven ganancias en la adquisición de conocimiento". La Encuesta de Actitudes de Lectura en Línea y Comportamientos y Habilidades (SORAB), busca satisfacer las variables afectivas en los estudiantes con la investigación de nuevas alfabetizaciones, por ejemplo, contexto para Internet (Ia mayoría de mis profesores me animan a investigar en Internet), actitudes generales de la computadora (es importante para mí poder utilizar un ordenador) y experiencia técnica (¿cuánto tiempo has estado accediendo a Internet?), con el fin de ser una evaluación general de las actitudes y comportamientos de los estudiantes hacia la lectura en línea (PUTMAN, 2014, p. 13). Considerando esto último, resulta relevante analizar las actitudes de los profesores de ciencias naturales (CCNN) y ciencias sociales (CCSS) hacia la enseñanza de competencias de consulta en línea y sus factores de fondo en el uso del Internet.

\section{Concepciones Generales y Componentes de Actitud}

Algunas definiciones describen actitudes como tener componentes afectivos y de sentimiento, cognitivos y de pensamiento o conocimiento y conductuales o de 
acción (KNEZEK; CHRISTENSEN, 2018). Según Hernández-Sampieri, Fernández-Collado y Baptista-Lucio (2010) la actitud es una predisposición aprendida, no innata, y estable aunque puede cambiar, a reaccionar de una manera valorativa, favorable o desfavorable, ante un objeto (individuos, grupos ideas, situaciones, entre otros). Koch (2005) señala que los sentimientos de los profesores y sus actitudes sobre la ciencia pueden afectar sentimientos y actitudes de sus alumnos. Dicho lo anterior, es que los datos son categorizaciones consistentes y características de un sujeto, durante cierto período relativas a objetos. Entre los métodos más utilizados para medir actitudes están: informes personales, técnicas proyectivas, formulación de opiniones, reacciones fisiológicas, escalas Likert, diferencial semántico y observaciones de conductas.

Conviene subrayar, que las respuestas pueden desarrollarse desde la manifestación de la conducta o a partir de las declaraciones verbales explicitas transformadas en respuestas. En concreto, cualquiera de estas respuestas puede usarse para inferir y deducir la actitud de un sujeto de estudio. No obstante, cada respuesta puede estar influenciada por diversas variables, disímil de la evaluación del objeto de actitud, lo cual esboza orientaciones teóricas complicadas. Además, las respuestas de un mismo sujeto de estudio con diferentes medidas de actitud pueden sugerir distintas actitudes subyacentes, así por ejemplo, un sujeto que efectúa afirmaciones verbales que no coinciden con la conducta que exhibe o con expresiones faciales espontáneas (MUÑOZ; QUINTANILLA; MANZANILLA, 2019).

\section{Competencia de Consulta en Línea (CCL)}

Las CCL se refieren a las actividades Web basadas en localizar, evaluar críticamente, sintetizar y comunicar información cuando el sujeto resuelve un problema con la ayuda de información en línea (SORMUNEN et al., 2017). Este enfoque se superpone con la investigación sobre alfabetismo de la información, pero la investigación se centra únicamente en las aptitudes necesarias en el entorno de Internet (SORMUNEN et al., 2017). Según Valverde-Crespo, Pro-Bueno y González-Sánchez (2018), las CCL resultan un conjunto de conocimientos, procedimientos y actitudes para buscar, seleccionar, evaluar y gestionar información de fuentes digitales e Internet, y transformarla en conocimiento para tomar decisiones y resolver problemas en contextos variados y emergentes a nivel personal y social. A su vez, las dimensiones de las competencias docentes digitales (RANGEL BACA, 2015, p. 241), categorizan las CCL en la dimensión informacional que tiene como concepto de este las habilidades necesarias para la búsqueda y gestión de la información dispuesta en distintas fuentes, soportes o lenguaje. En consecuencia, se pueden desarrollar competencias informacionales (alfabetización informacional), específicamente las relativas a investigación y consulta en línea, como por ejemplo: reconocer hechos, reconocer juicios, identificar ideas claves, discriminar entre fuentes, evaluar críticamente, sintetizar e integrar ideas y reconocer fuentes primarias en los respectivos dominios disciplinares (SORMUNEN et al., 2017).

\section{Metodología de la Investigación}

Se propuso analizar actitudes hacia la enseñanza de CCL en profesores de ciencias naturales y ciencias sociales de colegios públicos de la Región Metropolitana de Chile, 
asociados a factores de fondo y el uso del Internet. Se planteó un diseño cuantitativo y flexible, es decir, considerando enfoques cuantitativos y cualitativos para explicar con más detalle lo que ocurre (VASILACHIS, 2007). En este sentido, se hizo posible conocer las características más relevantes del grupo bajo estudio, como unidades de análisis que trabajan bajo una escala ordinal, ideal para un Análisis de Correspondencias Múltiple (ACM), técnica que aplicada sobre variables categóricas permite consolidar las respuestas recopiladas en función de un conjunto de afirmaciones; estas se representan dos dimensiones, visualizando así la posición relativa de todas las respuestas y su nivel de asociación entre ellas, (ABASCAL; GRANDE, 2017). Para obtener evidencia, se exhiben los siguientes aspectos operativos de diseño y análisis de campo:

- Construcción de un instrumento para recopilar información sobre las actitudes de CCL. En este sentido, se determinó la confiabilidad del instrumento para reflejar la exactitud y precisión con la que se obtendrán resultados similares si se aplica el mismo instrumento varias veces a los mismos sujetos (PALELLA; MARTINS, 2010). Dicho coeficiente se calculó haciendo uso del software estadístico SPSS en su versión 23, evaluando así la consistencia interna de las afirmaciones, siendo este bastante robusto, pues arrojó un valor de $81 \%$.

- Organización de información y construcción de la base de datos para su posterior análisis, considerando agrupamiento de datos a partir de nuevas escalas.

- Descripción general de la muestra utilizando porcentajes de frecuencia y diagramas sobre cada variable en estudio como: Acceso y uso de internet (A), formación docente para la enseñanza de competencias de consulta en línea ( $P, Q$ y $R)$ y actitudes hacia la enseñanza de competencias de consulta en línea (Ñ).

- Reducción de afirmaciones a través de un ACM con el fin de optimizar el rendimiento del instrumento definitivo conformado por 44 afirmaciones para dejar solo aquellas que muestran el mayor nivel de aplicabilidad de la variabilidad total, permitiendo consolidar los perfiles de actitudes de competencias de consulta en línea en los profesores.

\section{Instrumento y Muestra}

En este caso el cuestionario que se aplicó considera principalmente 10 preguntas cerradas, aunque en la primera parte se sumaron 3 preguntas abiertas. Las 13 preguntas son necesarias para reportar las características de la muestra, particularmente para responder los factores de fondo, como género, edad, educación profesional, experiencia docente, formación docente en CCL. Se utilizó para las preguntas cerradas la escala de Likert la cual, no mide en cuánto es más favorable o desfavorable una actitud, sino un escalonamiento de actitudes, (ANDER-EGG, 2004, p. 135). Es más apropiado recoger la información de esta manera debido a que es posible ordenar los datos en tablas y gráficos facilitando la interpretación y construcción de conclusiones.

Las Tablas 1, 2 y 3 corresponden a las afirmaciones para la dimensión acceso y uso de internet $(A)$, formación docente para la enseñanza de $C C L(P, Q$ y $R)$ y actitudes hacia la enseñanza de $C C L(\tilde{N})$, respectivamente. 
Tabla 1 - Dimensión A: acceso y uso de internet

\begin{tabular}{|c|l|}
\hline Código & \multicolumn{1}{|c|}{ Variable } \\
\hline A1 & Tengo un computador/tablet \\
\hline A2 & Tengo un teléfono inteligente \\
\hline A3 & En la escuela puedo usar un computador (o una tablets) \\
\hline A4 & Tengo un computador (o una tablet/teléfono inteligente) con acceso a Internet en la casa \\
\hline A5 & Tengo un teléfono inteligente con conexión a Internet \\
\hline A6 & En la escuela puedo usar computadores (o tablet, etc.) para acceder a Internet \\
\hline
\end{tabular}

Fuente: elaboración de los autores.

Tabla 2 - Dimensión P, Q y R: formación docente para la enseñanza de CCL

\begin{tabular}{|c|c|}
\hline Código & Variable \\
\hline P1 & $\begin{array}{l}\text { Durante mis estudios de pre-servicio de maestro fui educado para desarrollar en la enseñanza de las competencias } \\
\text { de consulta en línea (para utilizar las TIC e Internet en la enseñanza, guiar a los estudiantes en la búsqueda y } \\
\text { evaluación de la información web, ¿etc.)? }\end{array}$ \\
\hline P2 & ¿Qué tan satisfecho está usted con la formación formal recibida? \\
\hline Q1 & He desarrollado mi experticia en la enseñanza de las competencias de consulta en línea a través de mi tiempo libre \\
\hline Q2 & ¿Qué tan satisfecho estás de tus logros obtenidos a través de tus propios esfuerzos? \\
\hline R1 & $\begin{array}{l}\text { ¿Mi comunidad escolar me ha apoyado en el desarrollo profesional en cuanto al uso de las TIC e Internet, asi } \\
\text { como en las pedagogías de consulta en la web? }\end{array}$ \\
\hline AD & Años de docencia total \\
\hline AD-AÑOS $>5$ & Años de docencia total, más de 5 años \\
\hline AE-2<AÑOS $<5$ & Años de docencia en la escuela actual, más 2 años y menos de 5 años \\
\hline AE-AÑOS>5 & Años de docencia en la escuela actual, más de 5 años \\
\hline A & Años \\
\hline AGE (5) & Edad \\
\hline CS SS & Profesores que enseñan ciencias sociales \\
\hline CS NN & Profesores que enseñan ciencias naturales \\
\hline
\end{tabular}

Fuente: elaboración de los autores.

Tabla 3 - Dimensión Ñ: actitudes hacia la enseñanza de CCL

\begin{tabular}{|l|l|}
\hline \multicolumn{1}{|c|}{ Código } & \multicolumn{1}{c|}{ Variable } \\
\hline$\tilde{\text { N1 }}$ & Prefiero guiar la investigación de los estudiantes en Internet en vez de enseñarles en la forma tradicional \\
\hline$\tilde{\text { N2 }}$ & Me resulta útil usar artículos de Wikipedia como materiales de aprendizaje \\
\hline$\tilde{\text { N3 }}$ & Pienso que lo(a)s niño(a)s que no aprenden a utilizar Internet no desarrollan competencias importantes \\
\hline$\tilde{\text { N4 }}$ & Yo creo que el uso de Internet en la escuela hace que el aprendizaje sea más interesante \\
\hline$\tilde{\text { N5 }}$ & Prefiero usar la Internet cuando quiero saber sobre algo \\
\hline$\tilde{\text { N6 }}$ & Ser capaz de usar Internet en la enseñanza de mis asignaturas es importante para mí \\
\hline$\tilde{\text { N7 }}$ & Creo que es muy importante aprender a usar Internet para obtener información \\
\hline$\tilde{\text { N8 }}$ & Puedo encontrar información fiable en Internet debido a que mucho(a)s experto(a)s publican sus textos allí \\
\hline$\tilde{\text { N9 }}$ & Me gusta Internet porque encuentro varias opiniones sobre preguntas interesantes para mí \\
\hline$\tilde{\text { N10 }}$ & Me gusta discutir y compartir mis ideas en Internet (por ejemplo, en el chat, WhatsApp, etc.) \\
\hline AD & Años de docencia total \\
\hline AD-AÑOS $>\mathbf{5}$ & Más de 5 años de docencia total \\
\hline AE-2<AÑOS $<5$ & Años de docencia en la escuela actual, más 2 años y menos de 5 años \\
\hline A & Años \\
\hline AGE (5) & Edad \\
\hline C-SOCIALES & Profesores que enseñan ciencias sociales \\
\hline C-NATURALES & Profesores que enseñan ciencias naturales \\
\hline
\end{tabular}

Fuente: elaboración de los autores. 
Cada dimensión consta de afirmaciones según las escalas de valoración presentadas como: afirmaciones desde $A 1$ hasta $A 6(S i=1$ y No = 2), afirmaciones P1, P2, Q1, Q2, R1 y desde Ñ1 hasta Ñ10 (Muy en desacuerdo $=1$, En desacuerdo $=2$, Ni de acuerdo ni en desacuerdo $=3$, De acuerdo $=4$, Muy de acuerdo $=5$ ). Respecto al instrumento es importante destacar que fue adaptado a partir de la Encuesta de Actitudes de Lectura en Línea y Comportamientos y Habilidades (SORAB) de Putman (2014) para luego traducirlo al finlandés y español. Fue validado y piloteado en el contexto del Proyecto AKA EDU 03 (2016-2019), que pretende mejorar el aprendizaje y la enseñanza competencial del conocimiento escolar en varios dominios a través de la consulta en línea del profesorado y el estudiantado de primaria. Por su parte, el cuestionario definitivo con 44 afirmaciones fue administrado sobre una muestra intencional y no estadística de 33 profesores de la Región Metropolitana que previamente se inscribieron y participaron en los talleres desarrollados en el marco del proyecto, de los cuales 16 profesores enseñan ciencias naturales y 17 profesores enseñan ciencias sociales en enseñanza básica (nivel primario) de la Región Metropolitana de Chile.

\section{Resultados}

La primera parte se trata de un análisis de estadística descriptiva de la dimensión acceso y uso de Internet de los profesores de ciencias naturales y ciencias sociales (A). Luego, se explican los resultados obtenidos por los profesores mediante el análisis de correspondencia múltiple (ACM), en las dimensiones formación docente para la enseñanza de CCL ( $P, Q$ y $R)$ y actitudes hacia la enseñanza de CCL (Ñ).

\section{Estadística Descriptiva de la Dimensión A}

El 52\% de los encuestados pertenecen a Ciencias Naturales (CCNN) y el $48 \%$ restante a Ciencias Sociales (CCSS), el 94\% de los profesores de CCNN tienen jornada completa en su establecimiento educacional. En contraste un $88 \%$ de los profesores de CCSS tienen jornada completa en su colegio. Un $50 \%$ y $47 \%$ de los profesores de CCNN y CCSS respectivamente tienen más de 5 años ejerciendo la docencia en su colegio actual. En relación a la edad, los profesores de CCNN tienen un promedio de edad de 38 años ( $25 \%$ son mayores de 40 años). Por otra parte, los profesores de CCSS tienen un promedio de edad de 42 años, (59\% son mayores de 40 años). Por su parte, a partir de la información asociada al acceso a la tecnología de información e Internet en la escuela y en el hogar, por parte de los CCNN y CCSS, se construyó el siguiente gráfico. 
Gráfico 1 - Acceso a la tecnología de información e Internet en la escuela y en el hogar

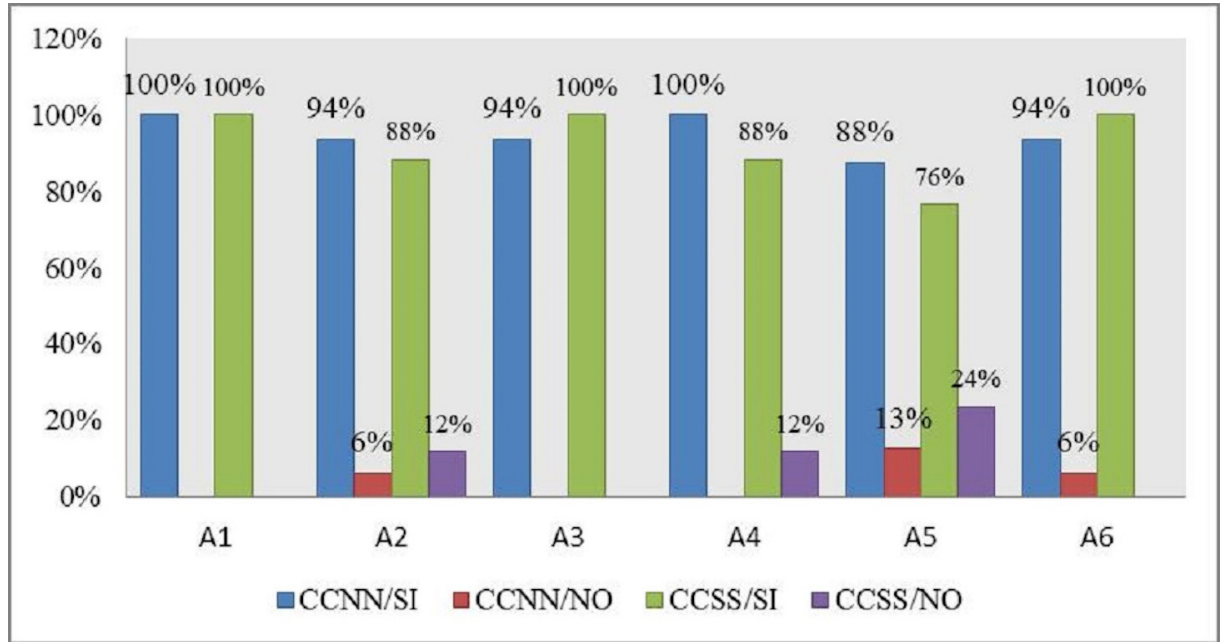

Fuente: elaboración de los autores.

El gráfico 1 evidencia que CCNN y CCSS, presentan un alto porcentaje de acceso a computador/tablet (A1), teléfono inteligente (A2) y en la escuela puede usar un computador o tablet (A3), todas las opciones superiores al $85 \%$. De igual forma, muestran un elevado acceso al Internet a través de las siguientes opciones, un computador (o una tablet /teléfono inteligente) en la casa (A4), teléfono inteligente (A5), o mediante un computador/tablet en la escuela (A6), cada una de las alternativas mencionadas presenta un valor mayor al $75 \%$.

\section{Análisis de Correspondía Múltiples}

Todos los análisis se realizaron a través del paquete estadístico SPSS V23, permitiendo consolidar un perfil de actitudes de los profesores de ciencias sociales (perfil CCSS) y ciencias naturales (perfil CCNN) hacia la enseñanza de las competencias de consulta en línea, tomando como referencia cada una de las dimensiones del instrumento. En efecto, se describe el set de datos en términos de nuevas variables no correlacionadas, esto a través de un ACM ejecutado en dos momentos, buscando según (ABASCAL; GRANDE, 2017) la mejor representación de los datos como puntos en un espacio euclídeo de baja dimensión, tratando de encontrar grupos entre las afirmaciones dispuestas. Los perfiles encontrados, presentan con claridad sus características en función de la variación de sus niveles en los porcentajes frecuenciales asociados a las cargas factoriales obtenidas del ACM.

\section{Dimensiones $\mathbf{P}, \mathbf{Q}$ y $\mathbf{R}$}

En el gráfico 2 en el perfil CCSS los profesores tienen menos de 2 años de ejercicio o más de 5 en una misma institución y, al menos 2 años de carrera, de jornada completa, con edades comprendidas entre 27 y 56 años, bastante diverso. Sobre la Formación docente para la enseñanza de las CCL, los profesores de CCSS tienen respuestas diversas, mostrando que muchos durante sus estudios de maestro fueron educados 
para la enseñanza de las CCL (específicamente para utilizar las TIC e Internet en la enseñanza, guiar a los estudiantes en la búsqueda y evaluación de la información web, etc.) (P1), mientras otros no, quedando el primero, bastante satisfecho con la formación formal recibida (P2), en oposición al segundo. Con relación a su Formación Informal, todos coinciden en haber desarrollado su experticia en la enseñanza de las CCL en su tiempo libre (Q1), a partir de esfuerzos propios, independientemente de haber recibido formación durante sus estudios (Q2). Finalmente, sobre el apoyo de la comunidad escolar esta ha sido bastante amplia en su desarrollo profesional, particularmente en cuanto al uso de las TIC e Internet y las pedagogías de consulta en la web (R1).

Gráfico 2 - Perfil CCSS encontrado sobre las dimensiones $\mathrm{P}, \mathrm{Q}$ y $\mathrm{R}$

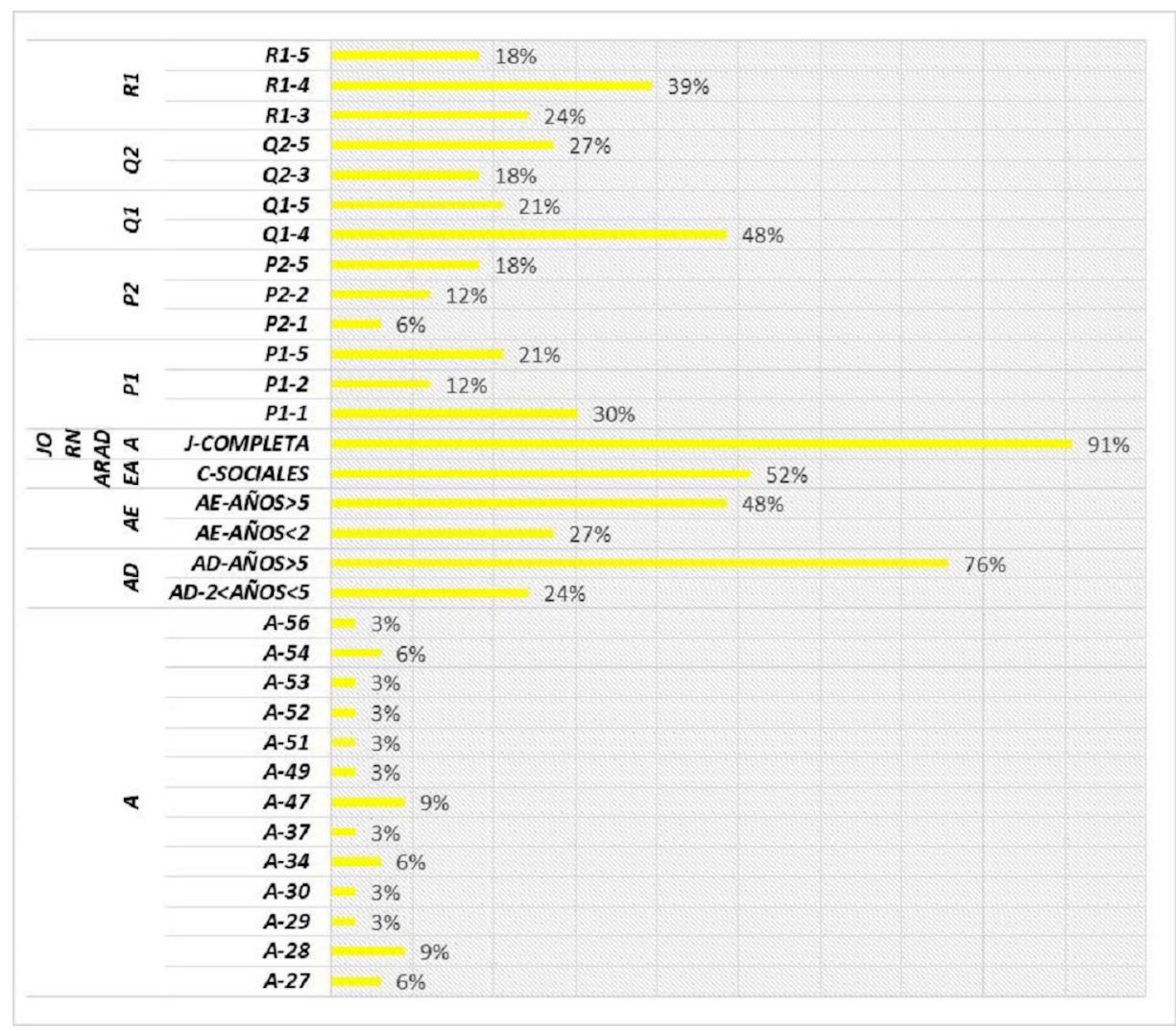

Fuente: elaboración de los autores.

Para el perfil CCNN (gráfico 3) los profesores tienen entre 2 y 5 años de ejercicio en una misma institución, con jornada completa mayormente (solo el $9 \%$ tiene jornada parcial), con edades comprendidas entre 31 y 61 años, bastante similar al grupo anterior en este aspecto. En relación con la formación docente para la enseñanza de las $\mathrm{CCL}$, un número significativo de profesores de CCNN durante sus estudios fueron educados para la enseñanza de las CCL (específicamente para utilizar las TIC e Internet en la enseñanza, guiar a los estudiantes en la búsqueda y evaluación de la información web, etc.) (P1), quedando satisfechos con la formación formal recibida (P2). Por otro lado, respecto a su Formación Informal indican que desarrollaron su experticia en la enseñanza de las CCL en su tiempo libre (Q1), por ende, a través de sus esfuerzos propios (Q2). Finalmente, sobre el apoyo de la comunidad escolar este no ha estado presente a en su desarrollo profesional, particularmente en cuanto al uso de las TIC e Internet y las pedagogías de consulta en la web (R1). 
Gráfico 3 - Perfil CCNN encontrado sobre las dimensiones P, Q y R4

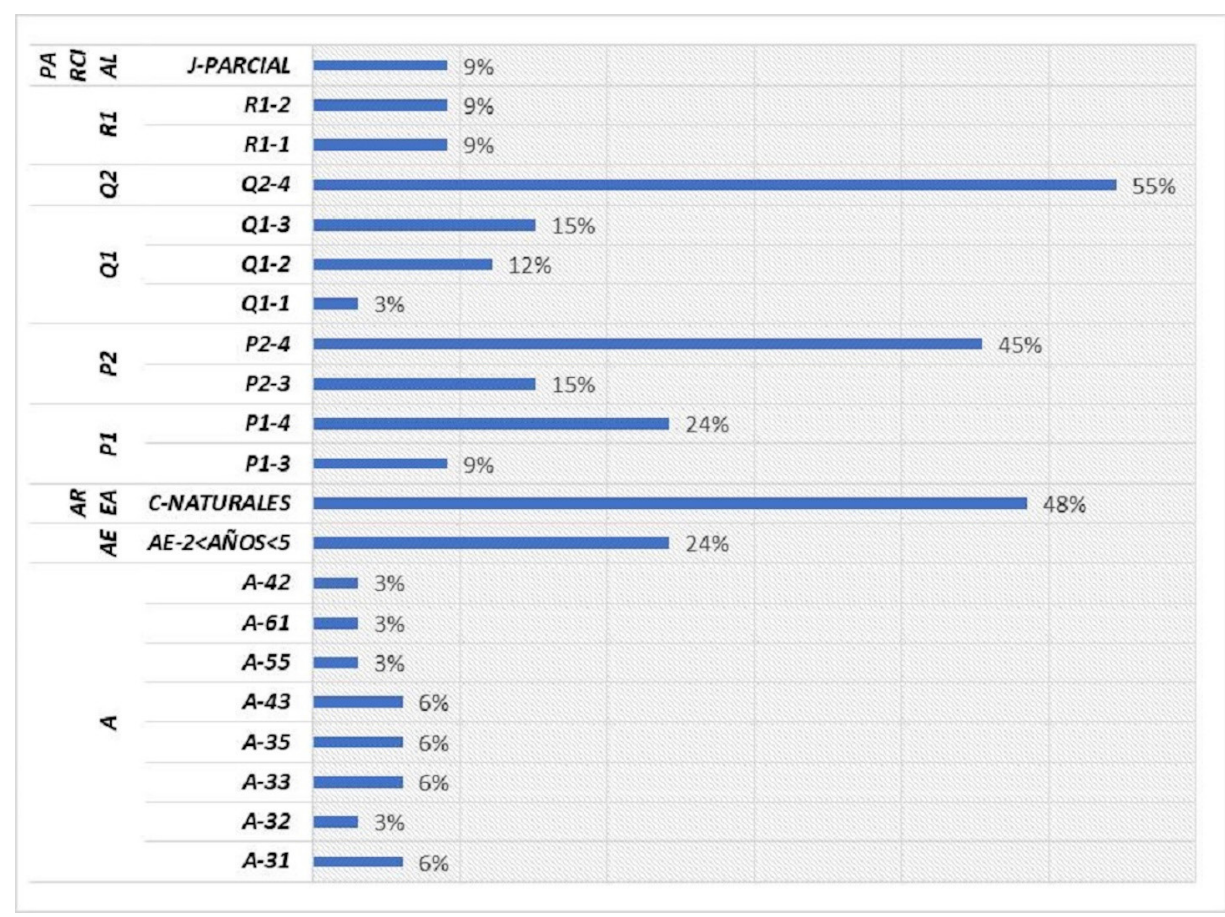

Fuente: elaboración de los autores.

\section{Dimensión Ñ}

En el gráfico 4 en el perfil CCSS los profesores tienen menos de 2 años de ejercicio o más de 5 en una misma institución y, al menos 2 años de carrera, de jornada completa, con edades comprendidas entre 27 y 56 años, bastante diverso. Sobre las actitudes hacia la enseñanza de CCL, los profesores de CCSS están parcialmente de acuerdo con preferir guiar la investigación de los estudiantes en Internet en vez de enseñarles en la forma tradicional (Ñ), pudiendo tal vez resultarles útil usar artículos de Wikipedia como materiales de aprendizaje (Ñ2), considerando parcialmente que lo(a)s niño(a) s que no aprenden a utilizar Internet no desarrollan competencias importantes (Ñ3), también que el uso de Internet en la escuela apunta a un aprendizaje más interesante (Ñ), teniendo preferencia en su uso al querer saber sobre algo (Ñ5), por tanto, la ven como una herramienta importante para el desarrollo de la asignatura (Ñ), aunado a la necesidad de aprender a usar Internet para obtener información (Ñ), sabiendo que mucha de ella es fiable debido a que está asociada a los textos de expertos publicados allí (Ñ8). 
Gráfico 4 - Perfil 1 encontrado sobre la dimensión Ñ

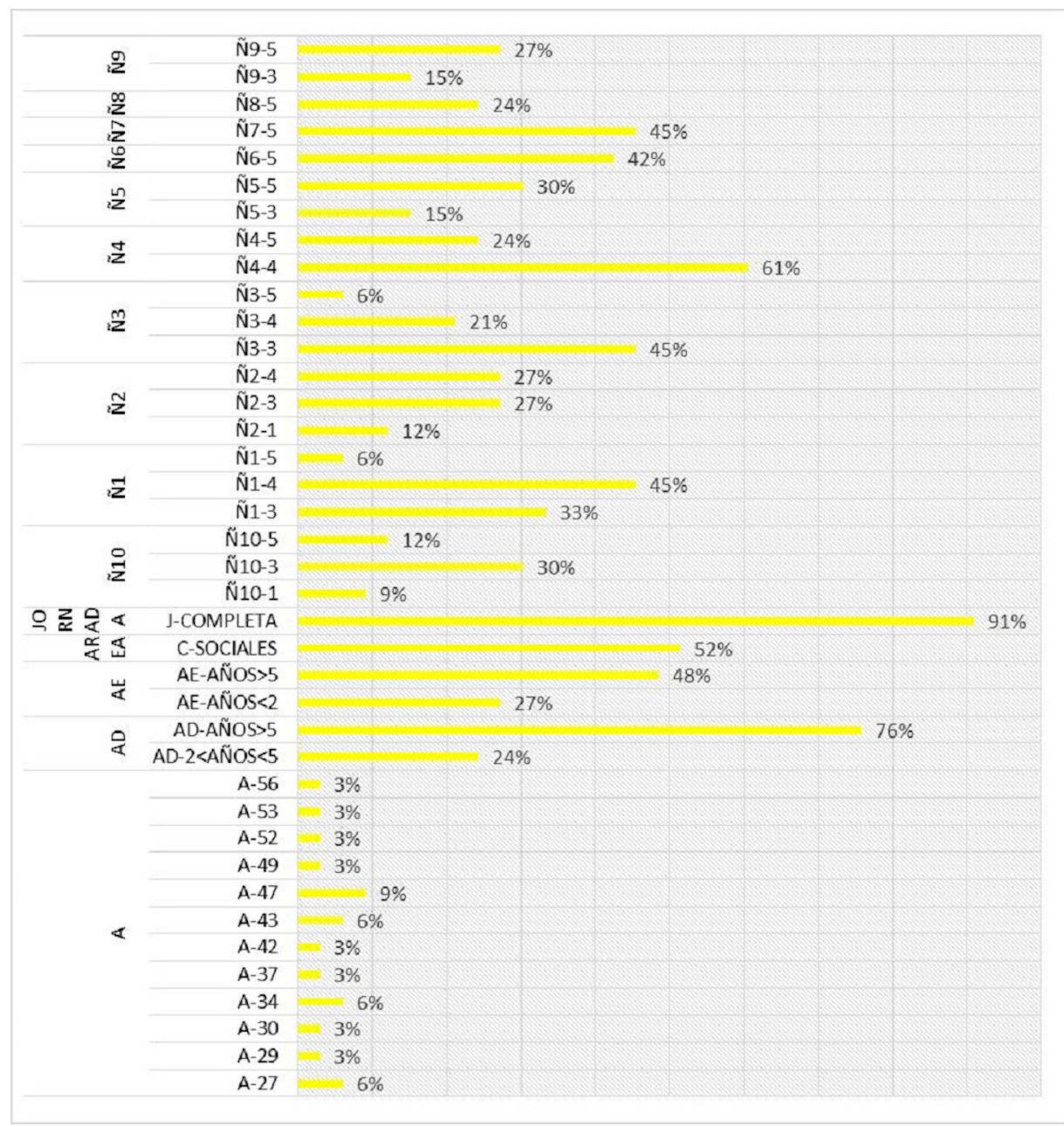

Fuente: elaboración de los autores.

Finalmente, manifiestan su gusto por Internet como herramienta para encontrar diversidad de opiniones sobre preguntas interesantes para el desarrollo de sus asignaturas (Ñ), permitiéndoles discutir y compartir ideas a través de la red con otros colegas (por ejemplo, en el chat, WhatsApp, etc.) (Ñ10).

Para el perfil CCNN (gráfico 5) los profesores tienen entre 2 y 5 años de ejercicio en una misma institución, con jornada completa mayormente (solo el $9 \%$ tiene jornada parcial), con edades comprendidas entre 28 y 61 años, bastante similar al grupo anterior en este aspecto. En relación con las actitudes hacia la enseñanza de CCL los profesores de CCNN están en desacuerdo con preferir guiar la investigación de los estudiantes en Internet en vez de enseñarles en la forma tradicional (Ñ1), resultándoles poco útil usar artículos de Wikipedia como materiales de aprendizaje (Ñ2), pensando que no necesariamente lo(a)s niño(a)s que no aprenden a utilizar Internet no desarrollan competencias importantes (Ñ3), no estando ni de acuerdo ni en desacuerdo con que el uso de Internet en la escuela apunta a un aprendizaje más interesante (Ñ), prefiriendo usar Internet solo al investigar para sí mismo (Ñ), siendo entonces bastante importante para el desarrollo de su asignatura(Ñ), es decir, es una herramienta útil para él pero no para el estudiante, pues no tiene dominio suficiente para aprovecharla. Por tanto, considera relevante aprender a usar Internet para obtener información (Ñ7), sobre todo teniendo certeza de que mucha de ella es fiable, pues proviene de los textos publicados allí por expertos (Ñ). 
Gráfico 5 - Perfil CCNN encontrado sobre la dimensión Ñ

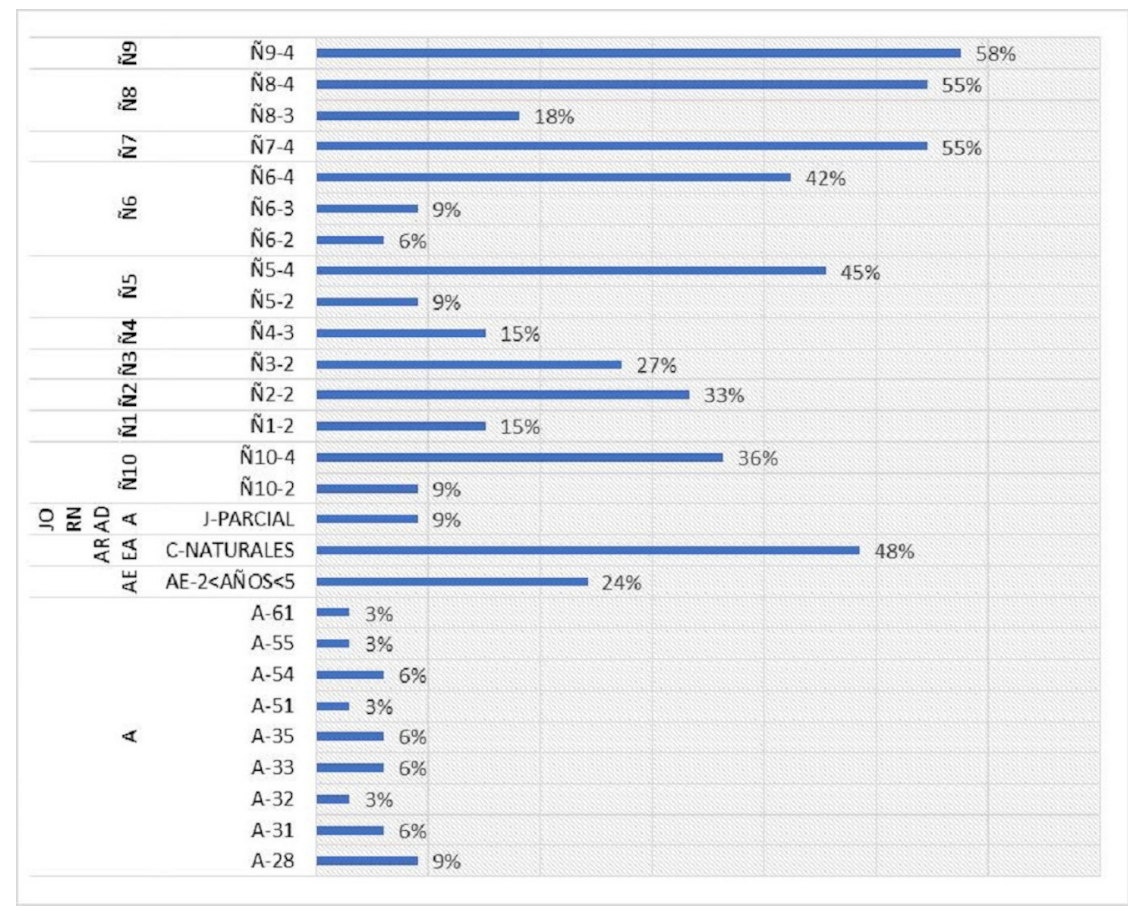

Fuente: elaboración de los autores.

\section{Discusión y Conclusiones}

En relación con la edad y experiencia docente debido a que los profesores de ciencias sociales son (59\%) mayores de 40 años. Por el contrario, solo el $25 \%$ de los profesores que enseñan ciencias naturales son mayores de 40 años. Estos resultados se pueden relacionar con los estudios planteados por Valdés Cuervo et al. (2011) y Yilmaz y Bayraktar (2014), en la cual los profesores de un rango superior a los 40 años no tienen una actitud tan efectiva hacia la enseñanza de las competencias de consulta en línea, debido a que reducen sus conocimientos informáticos y esto conlleva a una menor periodicidad de uso de las TIC.

Por otra parte, los profesores expresaron estar satisfechos con su formación formal, el $75 \%$ (CCNN) y 53\% (CCSS). Según el último resultado, se puede deducir que la formación inicial y permanente de los profesores de CCSS fue insuficiente e instrumentalista en el uso de las nuevas tecnologías para la enseñanza de las CCL, ya que más del $80 \%$ de ellos manifestaron que su experticia en la enseñanza de estas se debe a su formación informal. Esto coincide con la investigación formalizada por Svensson y Baelo (2015), comprobando que las instituciones de formación docente de varios países no se ocupaban apropiadamente en mejorar las CCL de los profesores, y su formación es de uso instrumental de las computadoras e Internet. Además, las instancias de perfeccionamiento de las TIC son precarias y en las carreras de pedagogía no parece ser un requisito constituir un curso formal de TIC (KRUMSVIK, 2014). Con relación a la dimensión de acceso y uso del Internet (A), los CCSS indicaron que al menos una vez al día por menos de 2 horas, usan Internet para propósitos pedagógicos en la escuela y en la casa. Esto se relaciona con los estudios de Almerich et al. (2010), concluyendo que el Internet es el recurso tecnológico que más conoce y utiliza el profesorado, con el fin de obtener información para sus clases. 
Por su parte, en los profesores de CCNN el Acceso y uso de Internet tiende a ser alto, en esencia lo utilizan diariamente por al menos 2 horas y el $62 \%$ y $51 \%$ lo emplea para propósitos pedagógicos y tareas administrativas en la escuela, respectivamente.

En esto no difiere con los resultados del profesorado que enseña CCSS y también tiene directa relación con lo que menciona Almerich et al. (2010) y Cebrián y Pérez (2003), señalando que los profesores utilizan el Internet como fuente de información para el acceso de bases de datos y documentos. Ramírez, Cañedo y Linuesa (2012) reafirman lo mencionado anteriormente con respecto a los profesores de CCSS y CCNN, pues según sus resultados los profesores afirmaban estar de acuerdo con que los recursos ofrecidos en Internet resultan imprescindibles para la docencia. Según Cebrián y Pérez (2003), utilizan el Internet como medio para tareas usuales y en cierta medida tradicional, por tanto, solamente buscan motivar a los estudiantes y que accedan a más información.

Ambos grupos de profesores tienen un acceso diario y frecuente a Internet y lo utilizan principalmente para propósitos administrativos y pedagógicos. También declaran que el Internet es imprescindible para la docencia debido a que se puede utilizar como medio para tareas tradicionales y fuente de información para el acceso a bases de datos. Es fundamental que el profesor tenga una seguridad y actitud positiva hacia los beneficios permisibles del uso del Internet, debido a que según las referencias bibliográficas, sus creencias y actitudes constituyen la barrera final para una integración efectiva de las tecnologías en las salas de clases y el desarrollo de las CCL en los estudiantes. Se puede deducir que el profesorado de esta investigación utiliza de manera instrumental el Internet, para fines personales y la enseñanza de su asignatura, pero manifiesta que no es una herramienta tan útil para el estudiante, pues esto se puede deber a que no tiene dominio suficiente para aprovecharla.

Con relación a la dimensión de las actitudes hacia la enseñanza de CCL (Ñ), no existe un apoyo mayoritario por parte de los profesores de ambas asignaturas en relación con ser un guía para la investigación de los estudiantes utilizando Internet. Esto confirmaría que algunos la utilizan de forma tradicional en sus clases, solo para la preparación de material didáctico y de uso personal, por ejemplo, búsqueda de imágenes y vídeos para elaborar una estrategia de presentación con apoyo de proyección (fijas o multimedia), y no para promover la investigación de los estudiantes utilizando Internet. Su uso instrumental se puede relacionar con el trabajo de Schoech (2000); señala que al utilizar el Internet como herramienta de aprendizaje tiene la cualidad de ser flexible e interactivo, por ejemplo, el tiempo es más prolongado para la revisión voluntaria de los materiales y contenidos. También promueve la interacción inmediata con los miembros de algún grupo de trabajo y favorece compartir sus respectivos comentarios, ya que no están limitados por las condiciones físicas del aula tradicional. Asimismo, el 75\% de los profesores señalan que el uso de Internet en la escuela orienta un aprendizaje más interesante, teniendo preferencia en su uso al querer saber sobre algo, por tanto, la ven como una herramienta importante para el desarrollo de la asignatura. Esto se relaciona con lo postulado por Cebrián y Pérez (2003), mencionando que al navegar por Internet puede encontrarse con información que les puede servir para la enseñanza y el aprendizaje de un contenido específico de las ciencias sociales (sitios web, foros, simuladores, revistas especializadas, imágenes, vídeos, estadísticas, simulaciones, entre otros). 
Del mismo modo, toda la muestra declara que es necesario aprender a usar Internet para obtener información, sabiendo que mucha de ella es fiable debido a que está asociada a los textos de expertos que publican normalmente allí. Como se ha mencionado, los docentes utilizan frecuentemente el Internet como fuente de información para el acceso de bases de datos y documentos (ALMERICH et al., 2010; CEBRIÁN; PÉREZ, 2003).

En consecuencia a estas actitudes sobre el uso informativo del Internet, los estudios de Gómez Crespo et al. (2014) y Valverde-Crespo, Pro-Bueno y GonzálezSánchez (2014), mencionan que algunas actividades propuestas por los docentes en relación a la búsqueda de información en Internet no tienen un fin claro y se torna una acción instrumentalista, que podría conducir a una formación limitada e insuficiente del estudiante para iniciar una búsqueda de información, interpretar su contenido y valorar su fiabilidad. Por otra parte, hubo diferencias considerables en las actitudes de los docentes respecto a la utilidad de usar artículos de Wikipedia como material de aprendizaje. El 62 \% (CCNN) y 30\% (CCSS) no están de acuerdo con usar artículos de Wikipedia como material de aprendizaje.

Con relación con el uso de Wikipedia, Sormunen y Lehtiö (2011) señalan que esta enciclopedia libre se beneficia de una amplia variedad de referencias citadas y del trabajo de buscadores de información capaces de confiar en su información. Volviendo a los resultados de este estudio, los profesores de ciencias naturales toman más en cuenta la calidad y fiabilidad de los artículos de Wikipedia debido que cualquier persona puede modificar y editar la información.

En definitiva, gran parte de los profesores utilizan Internet de forma tradicional en sus clases, o sea solo para recopilar información y preparar material educativo, y no para promover la investigación de los estudiantes utilizándolo. Así, las actividades elaboradas por los profesores con relación a la búsqueda y evaluación de la información en Internet no tienen un objetivo interesante y torna un trabajo instrumentalista, que podría conducir a una formación limitada e insuficiente del estudiante para iniciar una búsqueda de información, interpretar su contenido y valorar su fiabilidad. En cuanto a la relación de las actitudes hacia la enseñanza de CCL con sus factores de fondo, específicamente género, edad, experiencia docente, asignatura que enseñan, educación profesional, apoyo de la comunidad escolar, práctica docente y formación en CCL de los profesores, solo la edad fue la característica relevante en la muestra, asociada a la predisposición de los profesores, por ende, a mayor edad menor predisposición hacia la enseñanza de CCL. En consecuencia, la edad es un factor fundamental relacionado a los patrones de respuestas encontrados en los docentes. Los profesores de mayor edad no se formaron con la tecnología, sino que debieron pasar por un proceso de adaptación tecnológica. Por lo tanto, entre los profesores que enseñan ciencias sociales y ciencias naturales existe una brecha digital generacional; los de mayor edad presentan más carencia de competencias técnicas y didácticas para el uso del Internet.

Finalmente, en la actualidad circula muchísima información falsa (fake news) relacionada con la pandemia y el SARS-CoV-2 en las redes sociales y los medios de comunicación, que resulta peligrosa, en algunas ocasiones, ya que podrían afectar a la salud y puede hacer que aumente la tasa de contagio y provocar muertes de personas (PÉREZ-DASILVA; MESO-AYERDI; MENDIGUREN-GALDOSPÍN, 2020). Dado a este contexto resulta relevante que los profesores promuevan en sus clases remotas el desarrollo 
de CCL para que los estudiantes verifiquen la fiabilidad de la información que circula en Internet. En dirección aquello esta investigación entrega aportes significativos y críticos hacia el desarrollo de un modelo pedagógico que proporcione las herramientas necesarias para el uso responsable y competente del mundo digital en tiempos de pandemia.

\section{Agradecimientos}

Los autores de este trabajo agradecen al Proyecto AKA EDU 03 y a la Comisión Nacional de Investigación Científica y Tecnológica de Chile (CONICYT).

\section{Referencias}

ABASCAL, E.; GRANDE, I. Fundamentos y técnicas de investigación comercial. 13. ed. Madrid: ESIC, 2017.

ALMERICH, G.; SUÁREZ, J. M.; BELLOCH, C.; ORELLANA, N. Perfiles del profesorado a partir del conocimiento de los recursos tecnológicos y su relación con el uso que hacen de estas tecnologías. Revista Complutense de Educación, Madrid, v. 21, n. 2, p. 247-269, 2010. Recuperado el 8 mayo 2020 de: https://bit.ly/328Acas.

ANDER-EGG, E. Métodos y tecnicas de investigacion social IV: tecnicas para la recogida de datos e informacion. Buenos Aires: Lumen Humanitas, 2004.

CABERO, J. Formación del profesorado universitario en TIC: aplicación del método Delphi para la selección de los contenidos formativos. Educación XX1, Madrid, v. 17, n. 1, p. 111-132, 2014. DOI: https://doi.org/10.5944/educxx1.17.1.10707.

CEBRIÁN, M.; PÉREZ, M. D. ¿Para qué utilizan los profesores Internet en la docencia? In: CEBRIÁN, M. (coord.). Enseñanza virtual para la innovación universitaria. Madrid: Narcea, 2003. p. 46-77.

GÓMEZ CRESPO, M. A.; CAÑAS CORTÁZAR, A. M.; GUTIÉRREZ JULIÁN, M. S.; MARTÍN-DÍAZ, M. J. Ordenadores en el aula: ¿estamos preparados los profesores? Enseñanza de las Ciencias Barcelona, v. 32. n. 2, p. 239-250, 2014. DOI: https://doi.org/10.5565/rev/ensciencias.939.

HERNÁNDEZ-SAMPIERI, R.; FERNÁNDEZ-COLLADO, C.; BAPTISTA-LUCIO, P. Metodología de la investigación. 4. ed. México: McGraw-Hill, 2010.

KIILI, C.; LAURINEN, L.; MARTTUNEN, M. Skillful internet reader is metacognitively competent. In: HIN, L.T.; SUBRAMANIAN, R. (ed.). Handbook of research on new media literacy at the K12 level: issues and challenges. 2. ed. New York: Igi Global., 2009. p. 654-668.

KNEZEK, G.; CHRISTENSEN, R. The evolving role of attitudes and competencies in information and communication technology in education. In: VOOGT, J.; KNEZEK, G.; CHRISTENSEN, R.; LAI, K.-W. (ed.). Second handbook of information technology in primary and secondary education. Cham: Springer, 2018. p. 239-253.

KRUMSVIK, R. J. Teacher educators' digital competence. Scandinavian Journal of Educational Research, Oslo, v. 58, n. 3. p. 269-280, 2014. DOI: https://doi.org/10.1080/00313831.2012.726273.

LEU, D. J.; FORZANI, E.; RHOADS, C.; MAYKEL, C.; KENNEDY, C.; TIMBRELL, N. The new literacies of online research and comprehension: rethinking the reading achievement gap. Reading Research Quarterly, Newark, v. 50. n. 1, p. 37-59, 2015. DOI: https://doi.org/10.1002/rrq.85.

MUÑOZ, D.; QUINTANILLA, M.; MANZANILLA, M. Construcción y validación preliminar de un instrumento de evaluación de actitudes hacia la clase de química para estudiantes de educación secundaria. Educación Química, México, v. 30, p. 121-135, 2019. Recuperado el 8 mayo 2020 de: http://revistas.unam.mx/index.php/req/article/view/65592. 
O'BYRNE, W. I.; MCVERRY, J. G. Measuring the dispositions of online reading comprehension: a preliminary validation study. In: $57^{\text {th }}$ YEARBOOK of the National Reading Conference. Oak Creek, WI: National Reading Conference, 2008. p. 362-375.

PALELLA, S.; MARTINS, F. Investigación cuantitativa. 3. ed. Caracas: Editorial Fedupel, 2010.

PÉREZ-DASILVA, J. Á.; MESO-AYERDI, K.; MENDIGUREN-GALDOSPÍN, T. Fake news y coronavirus: detección de los principales actores y tendencias a través del análisis de las conversaciones en Twitter. Profesional de la Información, León, v. 29, n. 3, p. 4-16, 2020. DOI: https://doi.org/10.3145/ epi.2020.may.08.

PRENDES, M.; GUTIÉRREZ, I. Competencias tecnológicas del profesorado en las universidades españolas. Revista de Educación, Madrid, v. 361, p. 196-222, 2013. Recuperado el 8 mayo 2020 de: https://cutt.ly/syvylKs.

PUTMAN, S. M. Exploring dispositions toward online reading: analyzing the survey of online reading attitudes and behaviors. Reading Psychology, London, v. 35, n. 1, p. 1-31, 2014. DOI: https://doi.org/10.1080/02702711.2012.664250.

RAMÍREZ, E.; CAÑEDO, I.; LINUESA, M. C. Las actitudes y creencias de los profesores de secundaria sobre el uso de Internet en sus clases. Comunicar, Huelva, v. 19, n. 38, p. 147-155, 2012. DOI: https://doi.org/10.3916/C38-2012-03-06.

RANGEL BACA, A. Competencias docentes digitales: propuesta de un perfil. Píxel-Bit, Sevilla, n. 46, p. 235-248, 2015. Recuperado el 8 mayo 2020 de: https://cutt.ly/Yyvy9rq.

SCHOECH, D. Teaching over the internet: results of one doctoral course. Research on Social Work Practice, Newbury Park, USA, v. 10, n. 4, p. 467 487, 2000. DOI: https://doi. org/10.1177/104973150001000407.

SORMUNEN, E.; LEHTIÖ, L. Authoring wikipedia articles as an information literacy assignment: copy-pasting or expressing new understanding in one's own words. Information Research, Sheffield, England, v. 16, n. 4, 2011. Recuperado el 8 mayo 2020 de: https://bit.ly/2V55zkL.

SORMUNEN, E.; GONZÁLEZ-IBAÑEZ, R.; KIILI, C.; LEPPÄNEN, P. H. T.; MIKKILÄ-ERDMANN, M.; ERDMANN, N.; ESCOBAR-MACAYA, M. Performance-based test for assessing students' online inquiry competences in schools. In: KURBANOGLU, S.; BOUSTANY, J.; SPIRANEC, S.; GRASSIAN, E.; MIZRACHI, D.; ROY, L. (ed.). Information literacy in the workplace: 5th European Conference, ECIL 2017, Saint Malo, France, September 18-21, 2017, revised selected papers. Cham: Springer, 2018. p. 673-682. DOI: https://doi.org/10.1007/978-3-319-74334-9_69.

SVENSSON, M.; BAELO, R. Teacher students' perceptions of their digital competence. Procedia: social and behavioral sciences, New York, v. 180, p. 1527-1534, 2015. DOI: https://doi.

org/10.1016/j.sbspro.2015.02.302.

VALDÉS CUERVO, A. A.; ANGULO ARMENTA, J.; URÍAS MARTÍNEZ, M. L.; GARCÍA LÓPEZ, R. I.; MORTIS LOZOYA, S. V. Necesidades de capacitación de docentes de educación básica en el uso de las TIC. Pixel-Bit, Sevilla, n. 39, p. 211-223, 2011. Recuperado el 8 mayo 2020 de: https://bit. ly/37HyX3g.

VALVERDE-CRESPO, D.; PRO-BUENO A.; GONZÁLEZ-SÁNCHEZ, J. La competencia informacionaldigital en la enseñanza y aprendizaje de las ciencias en la educación secundaria obligatoria actual: una revisión teórica. Revista Eureka sobre Enseñanza y Divulgación de las Ciencias, Cádiz, v. 15, n. 2, p. 2105-15, 2014. DOI: https://doi.org/10.25267/Rev_Eureka_ensen_divulg_cienc.2018. v15.i2.2105.

VASILACHIS, I. Estrategias de investigación cualitativa. Buenos Aires: Gedisa, 2007.

YILMAZ, O.; BAYRAKTAR, D. M. Teachers' attitudes towards the use of educational technologies and their individual innovativeness categories. Procedia: social and behavioral sciences, New York, v. 116, p. 3458-3461, 2014. DOI: https://doi.org/10.1016/j.sbspro.2014.01.783. 